\title{
Clinical Trial and In Vitro Study for the Role of Cartilage and Synovia in Acute Articular Infection
}

\author{
Elia R. Langenmair, ${ }^{1}$ Eva J. Kubosch, ${ }^{1}$ Gian M. Salzmann, ${ }^{1,2}$ \\ Samuel Beck, ${ }^{1}$ and Hagen Schmal ${ }^{1,3}$ \\ ${ }^{1}$ Department of Orthopedics and Trauma Surgery, Albert-Ludwigs University Medical Center Freiburg, Hugstetter Street 55, \\ 79106 Freiburg, Germany \\ ${ }^{2}$ Schulthess Clinic, Zurich, Lenghalde 2, 8008 Zurich, Switzerland \\ ${ }^{3}$ Department of Orthopaedics and Traumatology, Odense University Hospital and Department of Clinical Research, \\ University of Southern Denmark, Sdr. Boulevard 29, 5000 Odense C, Denmark
}

Correspondence should be addressed to Hagen Schmal; hagen.schmal@freenet.de

Received 11 July 2015; Revised 8 October 2015; Accepted 12 October 2015

Academic Editor: Onkar P. Kulkarni

Copyright (C) 2015 Elia R. Langenmair et al. This is an open access article distributed under the Creative Commons Attribution License, which permits unrestricted use, distribution, and reproduction in any medium, provided the original work is properly cited.

Objective. Osteoarthritis is a long-term complication of acute articular infections. However, the roles of cartilage and synovia in this process are not yet fully understood. Methods. Patients with acute joint infections were enrolled in a prospective clinical trial and the cytokine composition of effusions compared in patients with arthroplasty $(n=8)$ or with intact joints $(n=67)$. Cytokines and cell function were also analyzed using a human in vitro model of joint infection. Results. Synovial IL- $1 \beta$ levels were significantly higher in patients with arthroplasty $(p=0.004)$. Higher IL-1 $\beta$ concentrations were also found in the in vitro model without chondrocytes $(p<0.05)$. The anti-inflammatory cytokines IL-4 and IL-10 were consistently expressed in vivo and in vitro, showing no association with the presence of cartilage or chondrocytes. In contrast, FasL levels increased steadily in vitro, reaching higher levels without chondrocytes $(p<0.05)$. Likewise, the viability of synovial fibroblasts (SFB) during infection was higher in the presence of chondrocytes. The cartilage-metabolism markers aggrecan and bFGF were at higher concentrations in intact joints, but also synthesized by SFB. Conclusions. Our data suggest an anti-inflammatory effect of cartilage associated with the SFBs' increased resistance to infections, which displayed the ability to effectively synthesize cartilage metabolites.The trial is registered with DRKS 00003536, MISSinG.

\section{Introduction}

Although the treatment of synovial bacterial infections usually succeeds, long-term complications include the development of osteoarthritis (OA) [1]. Proinflammatory mediators are suspected to influence chondrocyte differentiation and survival. The role of chondrocytes in this process is not yet fully understood; however, there is growing evidence that cartilage plays an active role during inflammation and is not only target [2]. Furthermore, the interaction between synovia and chondrocytes seems to contribute to the balance of anabolic and catabolic processes associated with OA. Considering the capacity of synovia to regenerate and cartilage's limited capacity to do so, the relation between these two anatomical structures might be decisive in regulating repair processes [3]. The approach taken in this study is a combination of biomarker analysis done in patients with acute synovial infections and a previously described in vitro model for intraarticular infections [4]. To discriminate between cartilage effects, we compared patients with an intact joint to patients having a total endoprosthesis. In the latter group, the effects of cartilage on inflammatory processes are eliminated. This situation was simulated in vitro comparing the articular infection model in a setting with or without chondrocytes. Our analysis included aggrecan, an essential component of the extracellular matrix and a sensitive marker for OA progress. Furthermore, bFGF, a typical cartilage metabolite associated with repair processes, was assessed [5]. We measured IL-1 $\beta$ 
to characterize the degree of inflammation [6]. Inspired by evidence of cartilage's anti-inflammatory properties, we also investigated the role of cytokines such as IL-4, IL-10, and Fas ligand (FasL) playing a potential role in our models and in vivo. Furthermore, TGF $\beta$ levels were assessed dependent on the presence of chondrocytes. We hypothesized that cartilage would reveal immunomodulatory features and similar in vivo and in vitro patterns.

\section{Material and Methods}

2.1. Clinical Trial. As has already been published [4], a consecutive series of 75 patients treated between April 2011 and November 2012 presenting the clinical symptoms of bacterial joint infection were recruited for the prospective collection of joint fluid. All patients suffered from pain, swelling, effusion, and elevated inflammatory serological parameters (e.g., C-reactive protein). Effusion from 76 affected joints was included in the analysis. Infections of knee (75\%), hip (6.6\%), ankle (1.3\%), and shoulder (17.1\%) were included. The trial was registered (MISSinG, DRKS 00003536) and approved by the Ethics Board of the University of Freiburg (ANEK-FRBRG-50/11). All patients participating in this study provided their written consent. There were 4 other patients whom we intended to include, but they had to be excluded because of storage-protocol violation $(n=3)$ or sudden death because of fulminant lung embolism $(n=1$, no written consent). Effusion was taken during arthroscopy or preoperative puncture and immediately frozen. Specimens were stored in liquid nitrogen until analysis. $81 \%$ of isolated bacteria belonged to Staphylococcus species. Evaluation via the Kellgren-Lawrence-Score [7] based on conventional Xrays was done by 3 independent orthopedic surgeons (EL, $\mathrm{EJK}$, and HS), resulting in a consensus decision.

2.2. In Vitro Model. We employed the in vitro model of synovial infection as previously described [4]. Briefly, human chondrocytes (CHDR) were isolated from femoral heads with a Kellgren-Lawrence-Score $\leq 2$ obtained during hip arthroplasty following femoral neck fractures. Following collagenase digestion, cells were cultured in Ham's F-12 medium with 1\% FBS (Invitrogen, Karlsruhe, Germany) and 1\% penicillin/streptomycin (Invitrogen, Karlsruhe, Germany). Human synovial fibroblasts (SFB) were isolated from synovia biopsies obtained during knee operations with an arthrotomy applying a similar protocol involving collagenase digestion and using the same media composition as indicated for CHDR. Human peripheral blood mononuclear cells (MNC) were isolated from heparin-treated human whole blood by a Ficoll-Paque (Pharmacia, Piscataway, NJ, USA) gradient. For 3D coculture, CHDR were embedded into alginate beads. Then, the cells were assembled having the SFB in monolayer overlayed with the CHDR in alginate beads; MNC were separated in the same well by cell culture inserts with $1 \mu \mathrm{m}$ pore size (BD, Heidelberg, Germany). After 3-day preconditioning, the coculture was challenged with $250 \mathrm{ng} / \mathrm{mL}$ LPS (Product number L6529, serotype E. coli 055:B5, Sigma Taufkirchen, Germany) and/or $100 \mathrm{ng} / \mathrm{mL}$ PMA (Product number P1585, Sigma Taufkirchen, Germany). Similar to previously reported data [4], the inflammatory reaction induced by LPS alone was limited and insufficient to reveal differential influences of the absence of cartilage. PMA alone had significant but fewer proinflammatory effects than the LPS and PMA combination; therefore, that data is not reported. Induction of inflammation by the significant overexpression of IL- $1 \beta$ was verified in each experiment. On days 3 and 7 , half of the media were exchanged to reduce the inflammatory burden mimicking a staged lavage as applied to treat in vivo joint infections. Media collected on days 0 , 3,7 , and 10 were preserved at $-80^{\circ} \mathrm{C}$ for analysis. Cocultures containing CHDR in alginate beads or not were compared in order to simulate joints with and without (arthroplasty) cartilage. The cells' preparation protocols were approved by the Ethics Committee of the University of Freiburg as part of the "Tissue Bank for Research in the Field of Tissue Engineering" project (GTE-2002) and the biobank "Osteo" (AN-EKFRBRG-135/14). Cells from the same donors were used when comparing different culture conditions. Lipopolysaccharides (LPS) are a cell-wall component of Gram-negative bacteria and provoke a distinct inflammatory stimulus including a potent activation of the macrophage secretory reaction [8]. While the LPS response is quickly diluted, a phorbol 12-myristate 13-acetate (PMA) application leads to stable, nontransient IL-1 accumulation [9]. Therefore, LPS and PMA are ideal substances to modulate the period and severity of an in vitro inflammation.

2.3. ELISA and Protein Content. Cytokine and protein concentrations (interleukin- [IL-] $1 \beta$, IL-4, IL-10, aggrecan [ACAN], basic fibroblast growth factor [bFGF], and Fas ligand $[\mathrm{FasL}]$ ) and transforming growth factor $[\mathrm{TGF}] \beta$ in synovial joint fluids from acutely infected knee joints or coculture supernatants were analyzed by ELISA (RnD, Minneapolis, MN, USA, and BioSource Deutschland GmbH, Solingen, Germany) according to the manufacturers' instructions. Briefly, this assay employs the quantitative sandwich enzyme immunoassay technique. The microplate was precoated with a specific monoclonal antibody. Supernatants were applied to the wells and, after washing, an HRPconjugated specific antibody was added to the wells. Following the next wash, color development was proportional to the protein concentration and calculated by comparison with a standard. A colorimetric method was applied to quantify total protein amount in the lavage fluids. The bicinchoninic acid (BCA) assay was available in kit form from Pierce (Rockford, IL, USA) and used according to the manufacturer's instructions [4]. All data from the analyzed cytokines and proteins are reported as relative expression to the total protein content. Statistical calculations were based on these values.

\subsection{CellTiter 96 AQueous One Solution Cell Proliferation} Assay (MTS Assay). Reagents were provided by Promega (Madison, WI, USA) and used according to the manufacturer's instructions [5]. MTS tetrazolium is bioreduced by cells into a colored formazan product that is soluble in tissue culture medium. Following a 10-day growth period, the reagent was added to each well of a microtiter plate. As per the manufacturer's instructions, we observed optical density at $490 \mathrm{~nm}$ after a 4 -h incubation period. 
2.5. Histology of SFB. SFB grown on cover slides in coculture approaches were fixed by PBS buffered 2\% formaldehyde for $24 \mathrm{~h}$ at room temperature, followed by Giemsa staining for $10 \mathrm{~min}$. Image acquisition and analysis were done by Zeiss Axioplan 200 and Axiovision 4.6, respectively [4].

2.6. Real Time PCR (RT-PCR). RNA samples from days 7 and 14 were transcribed into cDNA; RNA analysis was carried out for gene expression of TGF $\beta$. Total mRNA was prepared using the Qiagen RNeasy kit according to manufacturer's instructions (Qiagen, Hilden, Germany). Total RNA $(1 \mu \mathrm{g})$ was treated with 1 U DNAse I (Invitrogen, Karlsruhe, Germany) to remove genomic DNA. Poly-T primed cDNA synthesis was done using $1 \mathrm{U}$ reverse transcriptase III (RTIII, Invitrogen) per $1 \mu \mathrm{g}$ RNA according to manufacturer's instruction. TaqMan PCR assays were performed in 384well plates in Roche LightCycler480 (Roche, Mannheim, Germany) using the Roche LightCycler Mastermix. For gene expression analyses, Roche's universal ProbeLibrary Probes and recommended Universal ProbeLibrary Reference Gene Assays were used. The 2-step cycling conditions were denaturation: $95^{\circ} \mathrm{C}$ for $5 \mathrm{~min}$, followed by 45 amplification cycles: $95^{\circ} \mathrm{C}, 10 \mathrm{sec}, 60^{\circ} \mathrm{C}, 35 \mathrm{sec}$, and $72^{\circ} \mathrm{C}, 1 \mathrm{sec}$. Data was quantified via $\Delta \Delta \mathrm{CT}$ comparisons. Data were normalized by comparing genes of interest versus reference genes (GAPDH). Reaction efficiency is controlled by a relative standard curve and/or a calibrator per reaction. RT-PCR was done in triplicate; each value represents an average of 4 experimental preparations.

2.7. Data Analysis and Statistics. Concentrations of proteins and cytokines determined by the specific ELISAs and the BCA assay were calculated according to the manufacturers' instructions ( $\mathrm{RnD}$, Minneapolis, MN, USA; Thermo Scientific, Rockford, IL, USA), creating a standard curve and reducing data using a four-parameter logistic (4-PL) curve fit by using GraphPad Prism 5 software (GraphPad Software, Inc., La Jolla, CA, USA). All values were expressed as mean \pm standard error of the mean. Regarding the scores and all numerical values, statistical significance was tested nonparametrically primarily using the $U$-test according to Mann and Whitney. Numeric data sets were then further examined with one- and two-way analysis of variance, and individual group means were compared with the unpaired Student's $t$-test. In case of unequal variances, we applied the Aspin-Welch test. Incidences were compared using the Chi square test. Statistical significance was defined as $p<0.05$.

\section{Results}

3.1. Characterization of Patients. 75 patients with 76 articular infections were included in a prospective clinical trial. We compared the group with endoprostheses $(n=8)$ to the group with a normal joint $(n=68)$. Patients in both groups differed significantly according to certain clinical and epidemiological characteristics as shown in Table 1. In short, the postarthroplasty patients were older and presented more comorbidities and their diseases were more serious. The mean Kellgren-Lawrence-Score of the joints without endoprostheses was $1.6 \pm 0.26$, reflecting a mean moderate state of joint degeneration. In order to gain collectives with better comparability, patients with exclusive knee arthroplasties were separately analyzed $(n=6 / 51$, Table 1$)$. Although again clinical signs of disease were more serious, there was no statistically significant difference in age and the portion of isolated Staphylococcus species increased to $86 \%$ in this subgroup.

3.2. Total Protein Content. We detected no statistically significant difference in the total protein concentration in the inflamed joints with or without endoprosthesis $(0.057 \pm$ $0.014 \mathrm{~g} / \mathrm{mL}$ versus $0.048 \pm 0.003 \mathrm{~g} / \mathrm{mL}$ ). This was also apparent in vitro $\left(0.00178 \pm 2.85 \times 10^{-5} \mathrm{~g} / \mathrm{mL}\right.$ versus $0.00161 \pm$ $1.38 \times 10^{-4} \mathrm{~g} / \mathrm{mL}$ ) with no substantial variance during the culture period.

3.3. IL-1 $\beta$. According to calculations of all our patients' data, synovial IL-1 $\beta$ levels correlated statistically significantly with initial serological CRP concentrations (Spearman correlation coefficient $0.3, p=0.007$ ). The group of patients with endoprostheses revealed higher IL- $1 \beta$ levels than those with normal joints $\left(0.079 \pm 0.025 \times 10^{-6}\right.$ versus $0.015 \pm$ $0.004 \times 10^{-6}, p=0.004, U$-test, Figure 1(a)). This could be confirmed in the subgroup analysis of knee infections $(0.087 \pm$ $0.033 \times 10^{-6}$ versus $0.013 \pm 0.005 \times 10^{-6}, p=0.02$ ). This was also apparent in vitro; values peaked on day 3 in both groups $\left(0.050 \pm 0.001 \times 10^{-6}\right.$ versus $\left.0.026 \pm 0.002 \times 10^{-6}\right)$, declining thereafter. At all three measured time points after day 0, IL- $1 \beta$ was at higher concentrations in the cocultures without chondrocytes $(n=3$ per group, $p=0.0495$ at all time points, $U$-test, Figure $1(\mathrm{~b})$ ), demonstrating IL- $1 \beta$ induction via the inflammatory stimulus.

3.4. Aggrecan. In contrast to IL-1 $\beta$, the patient group with endoprostheses showed aggrecan levels that were lower than those with normal joints, although without attaining statistical significance $\left(3.13 \pm 1.14 \times 10^{-6}\right.$ versus $6.06 \pm 2.57 \times 10^{-6}$, $p=0.299$, Figure 2(a)). This was found for all patients and in the subgroup of knee infections. This was also shown in vitro; values increased over time in both groups, reaching the highest values on day $10\left(0.52 \pm 0.06 \times 10^{-6}\right.$ versus $2.52 \pm$ $\left.0.6 \times 10^{-6}\right)$. At all four measured time points, aggrecan was measured at higher concentrations in the cocultures with chondrocytes $(n=3$ per group, $p<0.05$ for all time points, $U$-test, Figure 2(b)), demonstrating aggrecan release beginning with the preculture period.

3.5. bFGF. bFGF concentrations were statistically significantly higher in the infected intact joints $(0.0063 \pm$ $0.0019 \times 10^{-6}$ versus $0.0195 \pm 0.0057 \times 10^{-6}, p=0.031$ [Aspin-Welch test], Figure 3(a), heterogeneous variances $[\mathrm{F}$ Test $\left.3.6 \times 10^{-6}\right]$ ). Although data of the subgroup with knee infections showed the same tendency, statistical analysis failed to confirm the significance of difference $(0.0063 \pm$ $0.0025 \times 10^{-6}$ versus $0.0190 \pm 0.0072 \times 10^{-6}$, n.s.). The in vitro data showed a time-dependent expression pattern for bFGF, with higher levels in the coculture with chondrocytes on day $3\left(0.0145 \pm 0.0014 \times 10^{-6}\right.$ versus $0.0995 \pm 0.0111 \times 10^{-6}, p=$ 
TABLE 1: Comparison of patients with arthroplasty and normal joints using epidemiological and inflammatory parameters. The first data set shows the comparisons for all patients, the second for the subgroup of patients with exclusive knee infections and knee arthroplasties. m: male, f: female, ASA: physical status according to the American Society of Anesthesiologists, ICU: intensive care unit, y: yes, n: no, and CRP: C-reactive protein in serum. ${ }^{*}$ Aspin-Welch test, ${ }^{+}$Chi square test, ${ }^{*} U$-test (Mann-Whitney), and ${ }^{++} t$-test.

\begin{tabular}{|c|c|c|c|}
\hline & Endoprosthesis $(n=8)$ & Normal joint $(n=68)$ & $p$ \\
\hline \multicolumn{4}{|l|}{ All patients } \\
\hline Age (years) & $71.3 \pm 3.4$ & $58.3 \pm 2.8$ & $0.001^{*}$ \\
\hline Gender $(\mathrm{m} / \mathrm{f})$ & $7 / 1$ & $44 / 24$ & n.s. ${ }^{+}$ \\
\hline ASA classification & $3.0 \pm 0.2$ & $2.2 \pm 0.1$ & $0.01^{\#}$ \\
\hline Body mass index & $31.6 \pm 1.6$ & $27.3 \pm 0.8$ & $0.03^{\#}$ \\
\hline ICU treatment $(\mathrm{y} / \mathrm{n})$ & $6 / 2$ & $16 / 52$ & $0.002^{+}$ \\
\hline Initial leucocyte count $(\mathrm{Tsd} / \mu \mathrm{L})$ & $13.7 \pm 1.9$ & $10.9 \pm 0.6$ & n.s. ${ }^{++}$ \\
\hline \multirow[t]{2}{*}{ Initial CRP $(\mathrm{mg} / \mathrm{L})$} & $154.9 \pm 38.1$ & $112.2 \pm 10.8$ & n.s. ${ }^{++}$ \\
\hline & Endoprosthesis $(n=6)$ & Normal joint $(n=51)$ & $p$ \\
\hline \multicolumn{4}{|c|}{ Subgroup with exclusive knee infections } \\
\hline Age (years) & $69.1 \pm 4.2$ & $55.9 \pm 3.3$ & n.s. ${ }^{*}$ \\
\hline Gender (m/f) & $5 / 1$ & $37 / 14$ & n.s. ${ }^{+}$ \\
\hline ASA classification & $3.0 \pm 0.3$ & $2.1 \pm 0.1$ & $0.02^{\#}$ \\
\hline Body mass index & $31.6 \pm 2.1$ & $27.1 \pm 0.9$ & n.s." \\
\hline ICU treatment $(\mathrm{y} / \mathrm{n})$ & $4 / 2$ & $12 / 39$ & $0.026^{+}$ \\
\hline Initial leucocyte count $(\mathrm{Tsd} / \mu \mathrm{L})$ & $13.0 \pm 2.5$ & $10.5 \pm 0.7$ & n.s. ${ }^{++}$ \\
\hline Initial CRP (mg/L) & $151.6 \pm 52.9$ & $103.0 \pm 11.9$ & n.s. ${ }^{++}$ \\
\hline
\end{tabular}

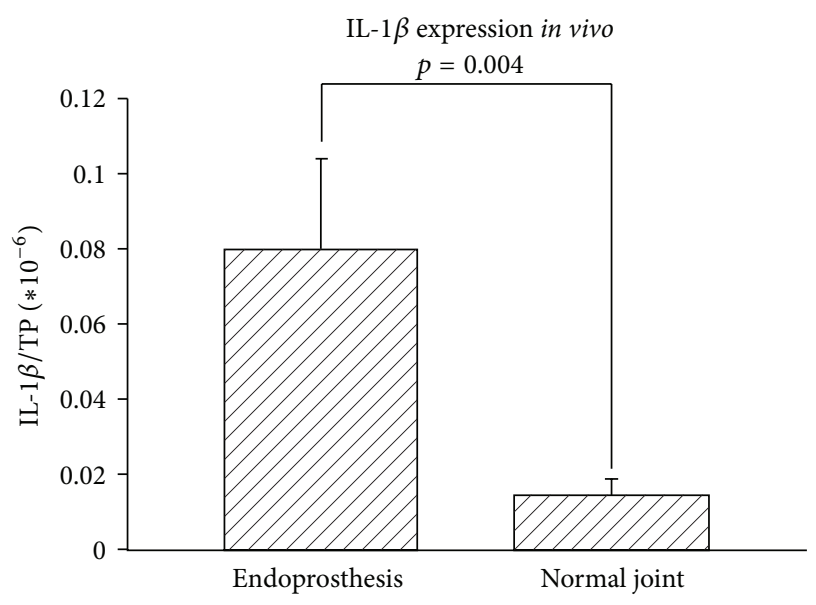

(a)

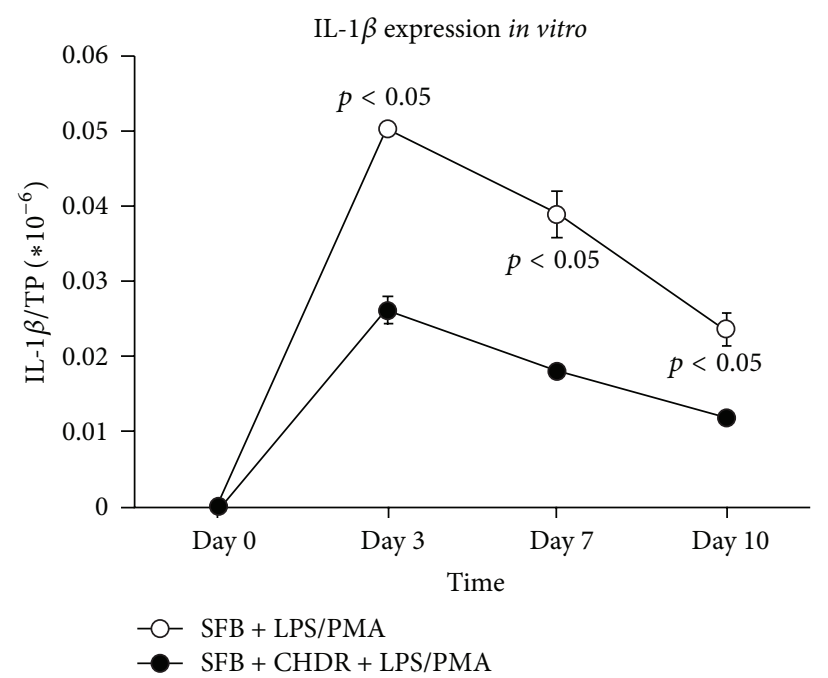

(b)

Figure 1: (a) shows that synovial IL-1 $\beta$ levels were statistically significantly higher in patients with an endoprosthetic implant (no cartilage) than in those with normal joints (with cartilage). (b) demonstrates the in vitro model's corresponding data. Similarly, IL-1 $\beta$ concentrations were statistically significantly higher in the setting without chondrocytes.

0.0495). We observed a reversal on day 7 , when the group without CHDR displayed higher levels $\left(0.54 \pm 0.07 \times 10^{-6}\right.$ versus $0.12 \pm 0.05 \times 10^{-6}, p=0.0495$, $U$-test, Figure 3(b)).

3.6. Anti-Inflammatory Mechanisms. IL-4, IL10, and FasL were examined for a possible role as anti-inflammatory cytokines. Although we detected all these proteins, none were differentially regulated in joints with or without endoprostheses (IL-4/TP $1.19 \pm 0.42 \times 10^{-7}$ versus $1.76 \pm 0.62 \times 10^{-7}$, IL$10 / \mathrm{TP} 2.4 \pm 0.89 \times 10^{-4}$ versus $4.8 \pm 2.6 \times 10^{-4}$, and FasL/TP $9.8 \pm 5.3 \times 10^{-7}$ versus $15.6 \pm 2.15 \times 10^{-7}$ ), which was also shown for the subgroup of knee infections. IL- 4 and IL-10 also demonstrated this in vitro, showing no significant differences at the given time points, and higher absolute IL-10 values than 


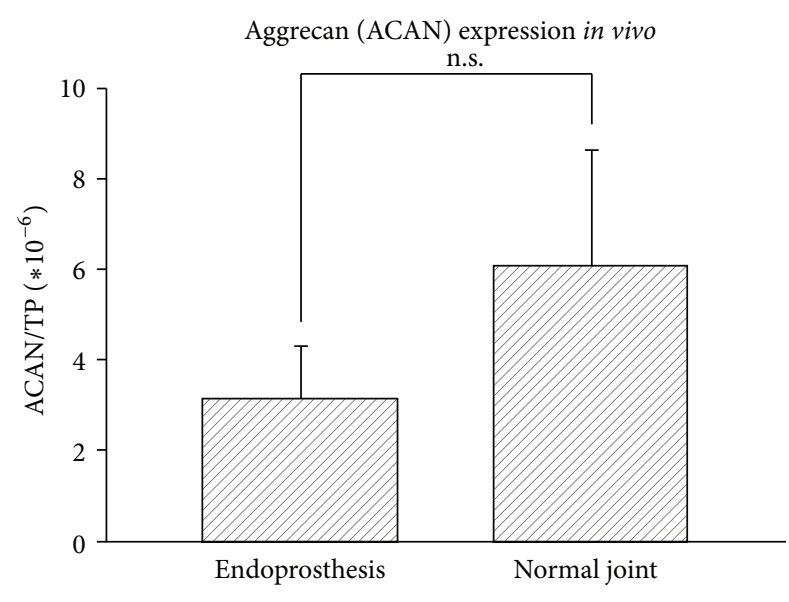

(a)

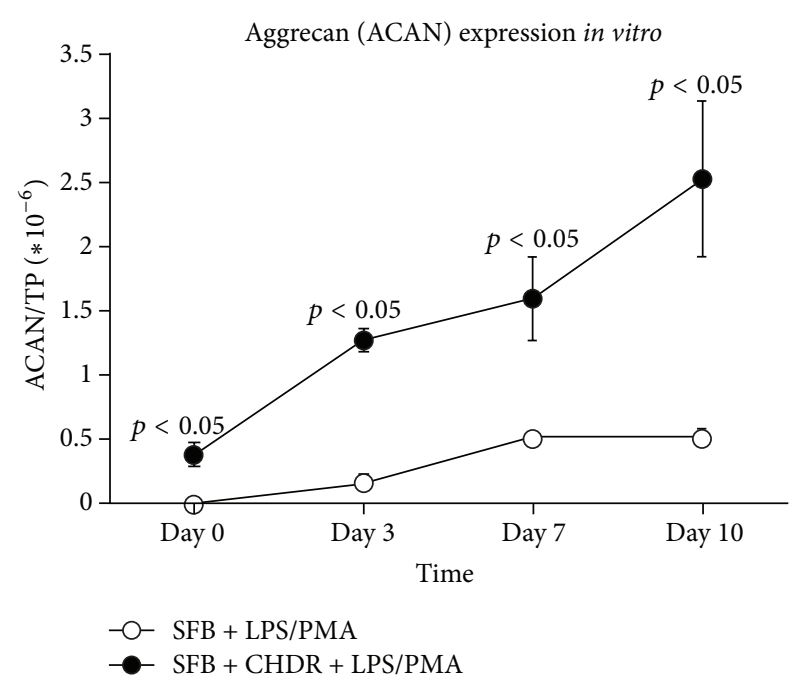

(b)

FIGURE 2: (a) shows that synovial aggrecan levels were reduced in patients with an endoprosthetic implant (no cartilage) compared to those with normal joints (with cartilage). This difference failed to attain statistical significance. (b) demonstrates the in vitro model's corresponding data. Similarly, aggrecan concentrations were statistically significantly higher in the setting with chondrocytes.

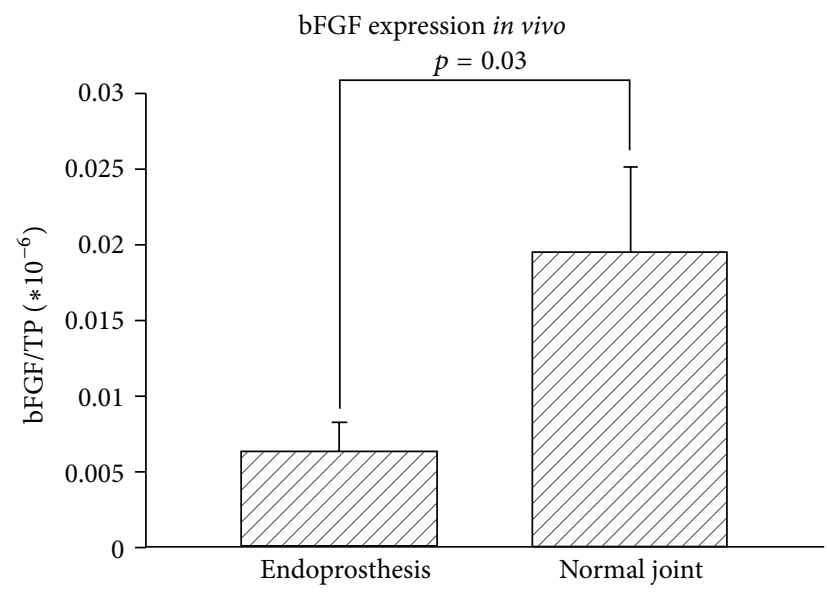

(a)

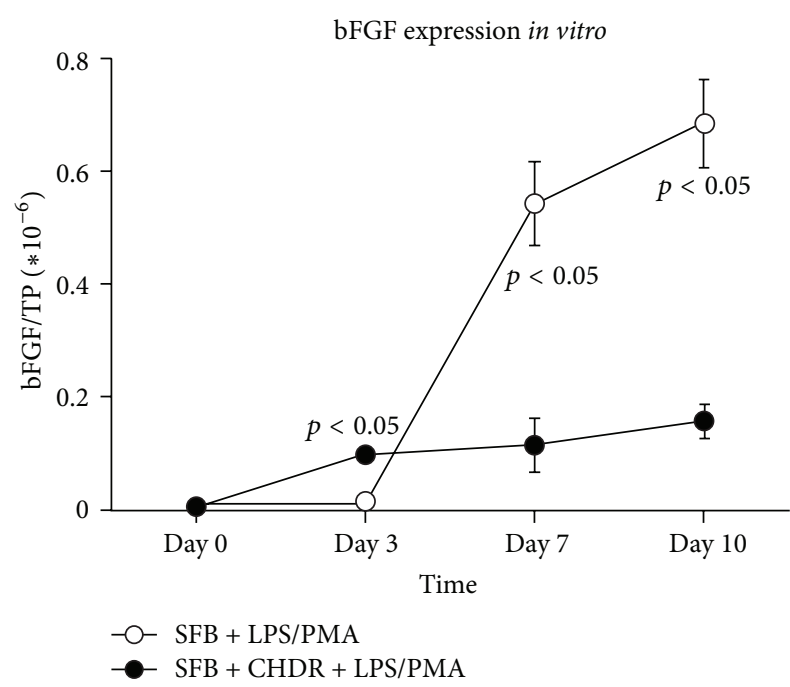

(b)

FIGURE 3: (a) shows that synovial bFGF levels were diminished in patients with an endoprosthetic implant (no cartilage) compared to those with normal joints (with cartilage). This difference reached statistical significance. (b) demonstrates the in vitro model's corresponding data; here, time-dependent regulation patterns revealed increasing bFGF concentrations in all groups over time. bFGF levels were higher in the group with CHDR on day 3, but lower on days 7 and 10 .

those noted in vivo for IL-4 (Figure 4(b)). FasL (Figure 4(a)) exhibited consistently rising levels in both groups, reaching higher concentrations in the coculture without CHDR on day $7(50.3 \pm 11.8 \mathrm{pg} / \mathrm{mL}$ versus $3.55 \pm 1.817 \mathrm{pg} / \mathrm{mL}$, $p=0.0495)$ and day $10(78.3 \pm 48.3$ versus $5.96 \pm 0.979$, n.s.).
3.7. SFB and CHDR Viability. To evaluate the viability of SFB, we carried out an MTS assay. While SFB cultured in the presence of CHDR demonstrated the same viability over the whole culture period under inflammatory conditions, SFB numbers decreased gradually in the absence of CHDR, falling to $25.8 \pm 8.5 \%$ (Figure $5(\mathrm{a})$ ). We confirmed this 


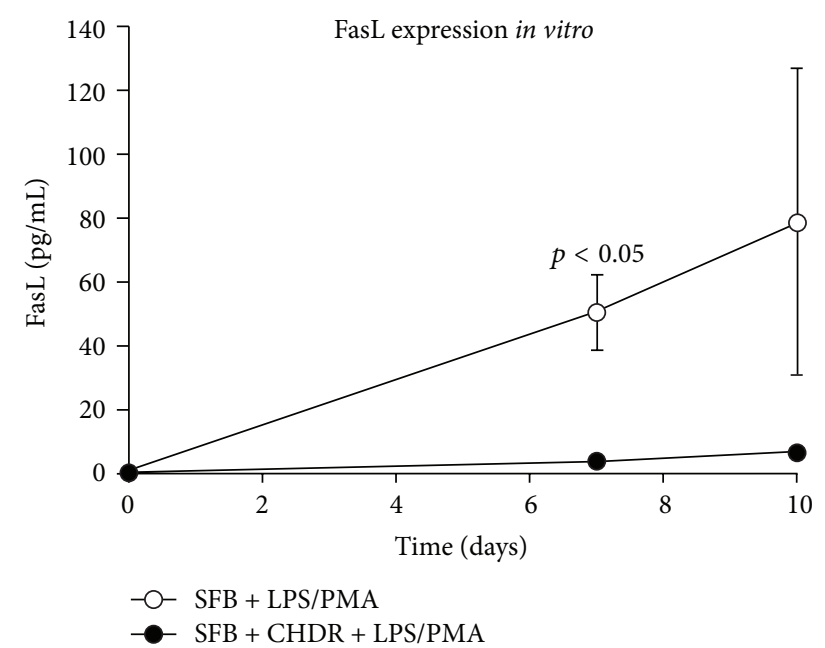

(a)

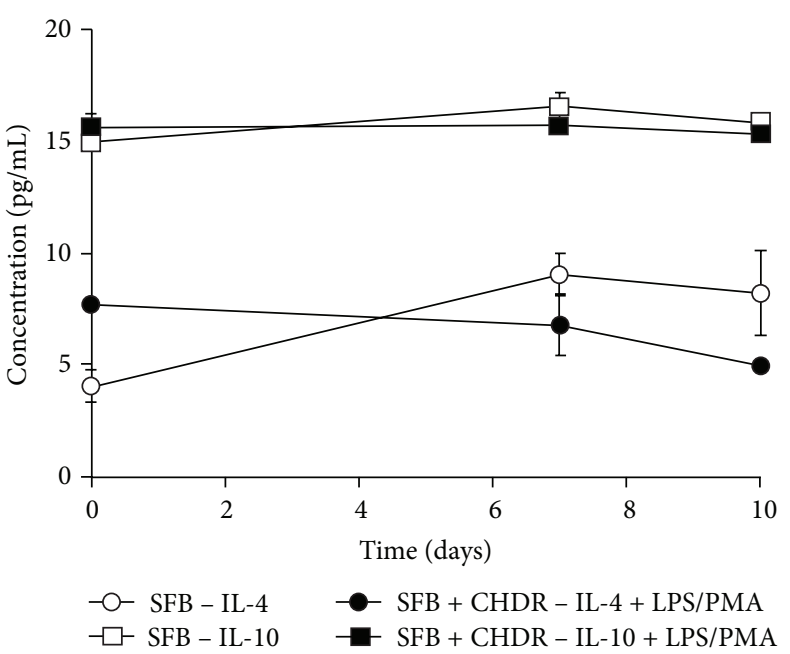

(b)

FIGURE 4: (a) shows FasL levels in the in vitro coculture. Concentrations increased over time in both groups but were statistically significantly more elevated in the group without CHDR. (b) illustrates IL-4 and IL-10 levels. We observed no dependency on time or presence of CHDR.

macroscopically via Giemsa staining for SFB cultures on cover slides (Figure 5(b)). During the coculture period, the metabolic activity of chondrocytes decreased slightly from day 7 to $72.8 \pm 0.4 \%$ on day 10 , a phenomenon that was slightly enhanced by adding LPS and PMA (59.2 $\pm 0.2 \%$, Figure 5(c)).

3.8. TGF $\beta$. The amount of TGF $\beta$ mRNA and protein was dependent on the presence of CHDR. The coculture with CHDR caused an upregulation of both TGF $\beta$ protein in the supernatant $(10.3 \pm 1.8 \mathrm{SFB}$ alone to $121 \pm 11 \mathrm{pg} / \mathrm{mL}$ with CHDR after 2 weeks) and TGF $\beta$ mRNA measured from SFB $(43.5 \pm 2.0$-fold SFB alone to $221 \pm 8.5$-fold TGF $\beta$ mRNA expression/GAPDH with CHDR after 2 weeks) when compared to SFB alone (Figure $6, p<0.05$ for protein and mRNA for both time points).

\section{Discussion}

This study's main finding is that cartilage exhibits antiinflammatory properties during acute articular infections, as demonstrated by decreased IL- $1 \beta$ expression in the presence of cartilage or CHDR in vivo and in vitro. Although CHDR increased the resistance of SFB to in vitro inflammatory conditions, the clinical trial's data does not allow definitive conclusions about the mechanisms behind the antiinflammatory effect. In vitro data suggest that FasL plays a key role, as it is in contrast to IL-4 and IL-10 increasingly secreted in the coculture during the inflammatory process. Furthermore, our results support the SFBs' capacity to synthesize typical representatives of cartilage metabolism such as aggrecan and bFGF. The production of these proteins was enhanced by inflammation.

Immunomodulation by cartilage has been described before, but until now the focus of in vitro studies had been on the ability of CHDR to synthesize specific cytokines such as IL-1 $\beta$ [2], bFGF, or aggrecan [4]. In addition, negative feedback reactions were described for human articular
CHDR attenuating inflammation by secreting soluble IL-1RA (sIL-1RA) in response to IL- $1 \beta$ and IL-6 [10]. This effect was induced by increased transcription from the sIL-1RA promoter. This mechanism was elucidated further, showing that IL- $1 \beta$ function in CHDR is inhibited by a peroxisome proliferator-activated receptor $\alpha$ pathway which functions by an increase in sIL-1RA production [11]. The inflammatory stimulus applied in the used in vitro model is mediated not only by inflammatory cytokines produced by MNC, but also directly via Toll-like receptors present on human articular CHDR [12]. There is evidence that the suppressive effects of LPS on cartilage biosynthetic activity depend on the presence of TLR- 4 and that the activation of this receptor is associated with IL- $1 \beta$ mRNA induction. The antianabolic effects mediated by TLR- 4 in CHDR may play a role in acute infection and may be antagonized by BMP-7 $[4,12]$. It is known that IL-1RA prevents interaction between IL-1 and its cell-surface receptors, thus competitively inhibiting the biological effects of IL-1 [13], but this does not fully explain antiinflammatory effects of cartilage (mirrored by the tendency to reveal lower systemic CRP-levels in our patients with intact joints). Therefore, we aimed to investigate whether other antiinflammatory mechanisms may be involved as upregulation of IL-4 and IL-10, as was recently suggested in association with treatment of acute lung injury or osteoarthritis [14, 15]. Our data support that both cytokines are expressed in vivo and in vitro, but neither of these proteins revealed an association with the presence or absence of cartilage. Recent studies have focused on FasL expression as another potential candidate for cartilage's anti-inflammatory effects. FasL expression by CHDR has been described as a possible mechanism to induce apoptosis in macrophages, thereby reducing the inflammation caused by cartilage grafts [16]. Furthermore, increased Fas and FasL levels were observed in the synovial fluid of patients with rheumatoid arthritis (RA) [17]. Data from that study [17] suggested that synovium modulates inflammation and proliferation via this 


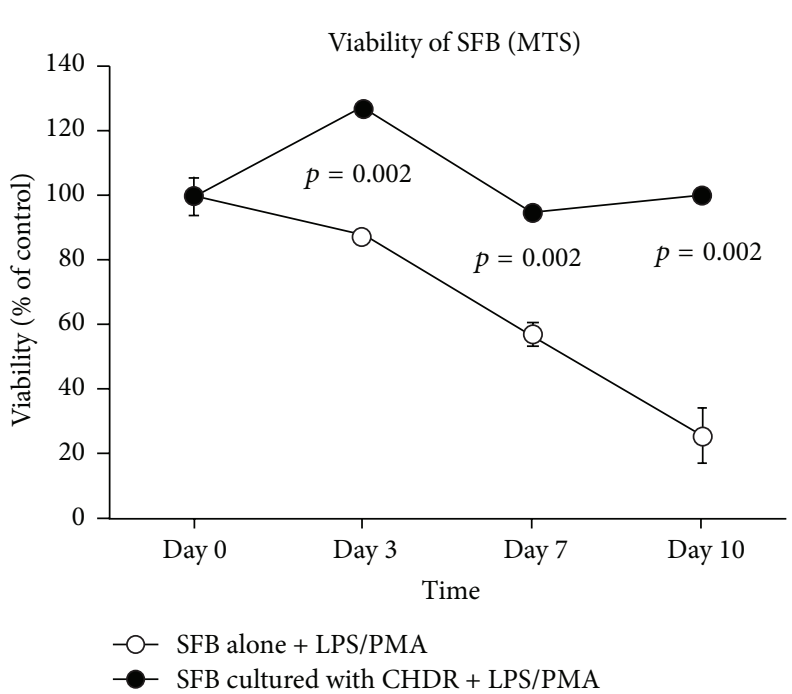

(a)

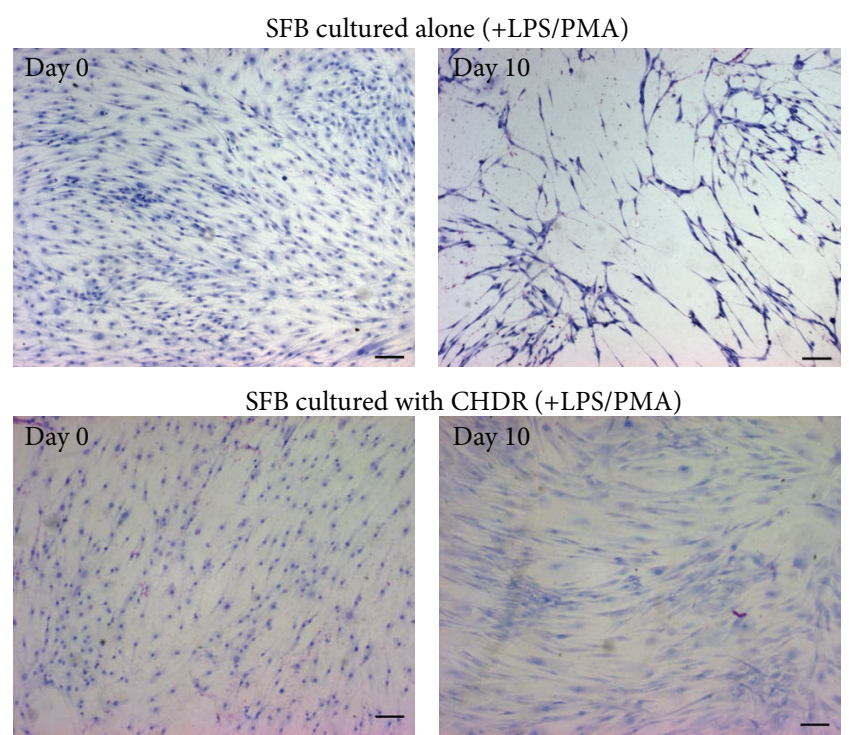

(b)

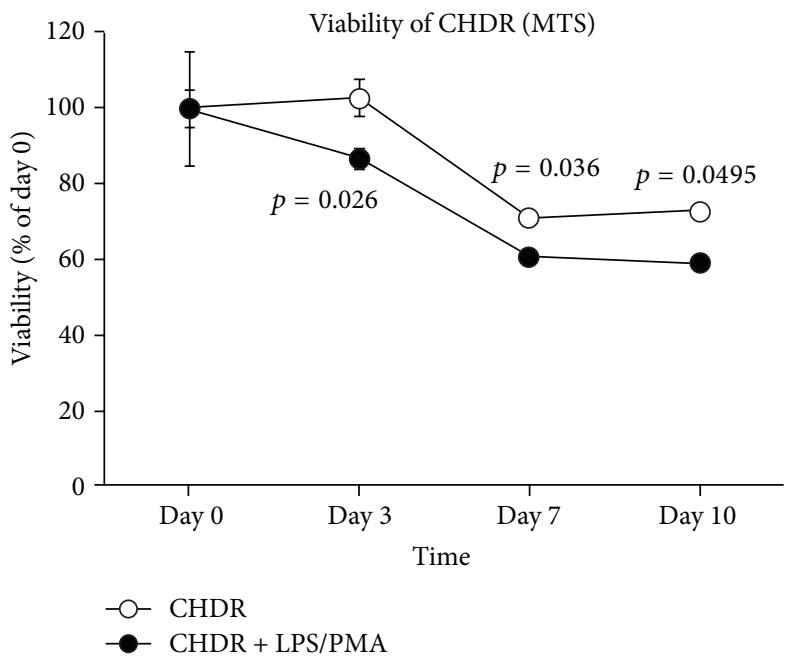

(c)

FIGURE 5: Viability of SFB was monitored using an MTS assay as shown in (a). While viability did not change over time in the presence of CHDR, a gradual decrease was observed during the incubation period in the absence of CHDR. (b) shows the correlating Giemsa staining for SFB cultures on cover slides confirming the MTS data. Scale bar $50 \mu \mathrm{m}$. The results of the MTS assay for CHDR in alginate beads are shown in (c). Metabolic activity slightly decreased from day 7 and was enhanced by adding LPS and PMA.

molecular mechanism. Our data confirm this, displaying FasL expression in the coculture and in joints with and without CHDR. One can only speculate about the reasons for significantly lower concentrations in the coculture with CHDR, but CHDR might be acting as a scavenger rather than an active producer in this setup. This hypothesis is supported by the fact that SFB did demonstrate higher viability during infection in the presence of CHDR. Although the metabolic activity of CHDR during the coculture decreased slightly over time, the influence of inflammatory conditions was less than that shown in conjunction with SFB, suggesting compensating mechanisms that result in greater resistance. Furthermore, the attenuation of inflammation by CHDR, for example, via sIL-1RA secretion, may also be the reason for less FasL secretion in the coculture medium.
As shown previously, the secretion of inflammatory cytokines as IL-1 $\beta$, monocyte chemoattractant protein(MCP-) 1, and IL-8 by chondrocytes depends on their maturity and is inversely regulated to TGF $\beta$ expression [18]. Tolllike receptors are expressed by mature chondrocytes, react to danger signals by production of further proinflammatory cytokines, and, therefore, support an inflammatory-driven cartilage degradation in a vicious circle [19]. The antagonistic effects of TGF $\beta$ and IL- 1 are mediated by regulation of TGF $\beta$ receptor type II and Smad7 [20] and are similar to the relation of proinflammatory TNF $\alpha$ and BMP-2, a member of the TGF $\beta$ superfamily [21]. Therefore, we hypothesized that $\operatorname{TGF} \beta$ might also mediate the immune-regulatory and antiinflammatory effect of chondrocytes. Although we have not directly demonstrated this, we were able to show induction 
TGF $\beta$ concentrations are increased in SFB supernatants in the presence of CHDR

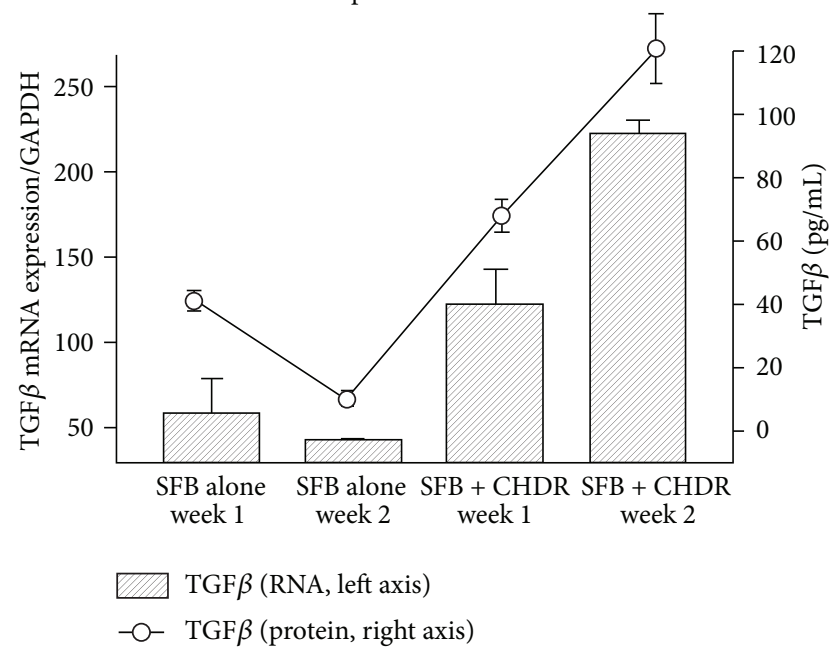

FIGURE 6: The coculture of SFB with CHDR caused an upregulation of both TGF $\beta$ protein and TGF $\beta$ mRNA $(p<0.05$ for both time points) compared to SFB alone.

of TGF $\beta$ mRNA in SFB by CHDR and increased TGF $\beta$ levels in the coculture of SFB and CHDR supporting a regulatory association.

Aggrecan has been identified as a reliable indicator for OA progression and matrix degradation [22] that is upregulated following injury or general inflammation. Both the breakdown of extracellular matrix (ECM) and enhanced synovial secretion are known to cause this effect $[23,24]$. The experimental data provided in this study confirm that both joint compartments, the synovium and cartilage, contribute to the aggrecan level increase we observed. Although the difference observed in the clinical trial between joints with and without cartilage did not reach statistical significance, we did note a tendency towards higher concentrations in the joints without arthroplasty. Furthermore, aggrecan levels rose steadily during the coculture in both groups, reaching higher concentrations in the presence of CHDR. Our understanding of the aggrecan's role has grown recently because it is not just an essential element of the ECM; it is also directly involved in immunoregulatory processes such as the induction of inflammation by HLA-B27-restricted epitopes [25].

bFGF (FGF-2) has been characterized as a chondrogenic mitogen typically involved in cartilage repair processes [5] demonstrating high potential in cartilage engineering [26]. Acute infection leads to increased synovial bFGF levels, but mRNA induction in CHDR is 10 -fold higher than in the SFB [4]. bFGF is secreted by CHDR released from ECM, enhances CHDR proliferation, induces a chondrogenic phenotype dependent on the experimental setting, and promotes articular cartilage repair by upregulating multiple growth factors such as BMPs and TGF $\beta$ [27]. Our data confirm this by demonstrating bFGF in the cavity of inflamed joints and gradually increasing concentrations in the in vitro model. Moreover, our results indicate that the potential of SFB to produce bFGF is remarkable and triggered by proinflammatory conditions. However, our clinical trial's data support that the main in vivo source is the cartilage itself. The time-dependent regulation pattern seen in vitro might be explained by the fact that bFGF is secreted by CHDR but may also be bound via receptors.

A critical evaluation of the presented in vivo data includes a comparison of both patient collectives. Naturally, they differ in the main discrimination criterion, the presence of an endoprosthesis, but they also had the same kind of clinical symptoms and in the subgroup analysis also the same effected joint with the predominantly same sort of causing bacteria. The groups differed in age, but based on a twin study no correlation between age and IL- $1 \beta$ expression could be found [28]. The same study reported a moderate heritability for IL-1 $\beta$ expression that declined with age due to an increase in unique environmental factors. Since the investigated population was rather old compared to the data published, the factor of heritability may be estimated to have a low influence. Therefore, both groups can be compared regarding the influence of the cartilage presence. Increased synovial IL- $1 \beta$ values should correlate with higher systemic inflammatory parameters. Despite not reaching statistical significance, the trend could be confirmed for leucocyte counts and plasma CRP-levels. This also plausibly explains the longer stay at an ICU, which really was statistically significant. Assuming a random distribution of other (unknown) parameters, a comparison based on the main discrimination criterion seems to be justified.

To summarize, we confirmed the hypothesis of cartilage's immunomodulatory properties. Acute infections induce processes that resemble OA development such as ECM degradation, the induction of cartilage repair cytokines, and the initiation of apoptosis. Generally speaking, cartilage would seem to exert a protective, anti-inflammatory effect that is at least partially associated with FasL action, resulting in the enhanced survival of SFB.

\section{Conflict of Interests}

The authors declare that there is no conflict of interests regarding the publication of this paper.

\section{Acknowledgments}

The study was funded by the Research Commission of the Albert-Ludwigs University Medical Center Freiburg and the Department of Education and Research Germany (01EC1001D).

\section{References}

[1] G. Mouzopoulos, V. C. Fotopoulos, and M. Tzurbakis, "Septic knee arthritis following ACL reconstruction: a systematic review," Knee Surgery, Sports Traumatology, Arthroscopy, vol. 17, no. 9, pp. 1033-1042, 2009.

[2] M. B. Goldring and M. Otero, "Inflammation in osteoarthritis," Current Opinion in Rheumatology, vol. 23, no. 5, pp. 471-478, 2011. 
[3] H. Schmal, I. H. Pilz, A. T. Mehlhorn et al., "Expression of BMPreceptor type $1 \mathrm{~A}$ correlates with progress of osteoarthritis in human knee joints with focal cartilage lesions," Cytotherapy, vol. 14, no. 7, pp. 868-876, 2012.

[4] I. H. Pilz, A. Mehlhorn, D. Dovi-Akue, E. R. Langenmair, N. P. Südkamp, and H. Schmal, "Development and retranslational validation of an in vitro model to characterize acute infections in large human joints," BioMed Research International, vol. 2014, Article ID 848604, 13 pages, 2014.

[5] H. Schmal, J. Zwingmann, M. Fehrenbach et al., "bFGF influences human articular chondrocyte differentiation," Cytotherapy, vol. 9, no. 2, pp. 184-193, 2007.

[6] B. D. Furman, D. S. Mangiapani, E. Zeitler et al., "Targeting pro-inflammatory cytokines following joint injury: acute intraarticular inhibition of interleukin-1 following knee injury prevents post-traumatic arthritis," Arthritis Research and Therapy, vol. 16, article R134, 2014.

[7] J. H. Kellgren and J. S. Lawrence, "Radiological assessment of osteo-arthrosis," Annals of the Rheumatic Diseases, vol. 16, no. 4, pp. 494-502, 1957.

[8] M. J. Auger, "Mononuclear phagocytes," British Medical Journal, vol. 298, no. 6673, pp. 546-548, 1989.

[9] M. J. Fenton, M. W. Vermeulen, B. D. Clark, A. C. Webb, and P. E. Auron, "Human pro-IL-1 beta gene expression in monocytic cells is regulated by two distinct pathways," The Journal of Immunology, vol. 140, no. 7, pp. 2267-2273, 1988.

[10] G. Palmer, P.-A. Guerne, F. Mezin et al., "Production of interleukin-1 receptor antagonist by human articular chondrocytes," Arthritis Research, vol. 4, no. 3, pp. 226-231, 2002.

[11] M. François, P. Richette, L. Tsagris et al., "Activation of the peroxisome proliferator-activated receptor $\alpha$ pathway potentiates interleukin-1 receptor antagonist production in cytokinetreated chondrocytes," Arthritis \& Rheumatism, vol. 54, no. 4, pp. 1233-1245, 2006.

[12] K. Bobacz, I. G. Sunk, J. G. Hofstaetter et al., “Toll-like receptors and chondrocytes: the lipopolysaccharide-induced decrease in cartilage matrix synthesis is dependent on the presence of tolllike receptor 4 and antagonized by bone morphogenetic protein 7," Arthritis and Rheumatism, vol. 56, no. 6, pp. 1880-1893, 2007.

[13] W. P. Arena, M. Malyak, C. J. Guthridge, and C. Gabay, "Interleukin-1 receptor antagonist: role in biology," Annual Review of Immunology, vol. 16, pp. 27-55, 1998.

[14] A. Lang, J. Neuhaus, M. Pfeiffenberger et al., "Optimization of a nonviral transfection system to evaluate Cox-2 controlled interleukin-4 expression for osteoarthritis gene therapy in vitro," The Journal of Gene Medicine, vol. 16, no. 11-12, pp. 352363, 2014.

[15] T. P. Shanley, H. Schmal, H. P. Friedl, M. L. Jones, and P. A. Ward, "Regulatory effects of intrinsic IL-10 in IgG immune complexinduced lung injury," Journal of Immunology, vol. 154, no. 7, pp. 3454-3460, 1995.

[16] Y. Fujihara, T. Takato, and K. Hoshi, "Macrophage-inducing FasL on chondrocytes forms immune privilege in cartilage tissue engineering, enhancing in vivo regeneration," Stem Cells, vol. 32, no. 5, pp. 1208-1219, 2014.

[17] D. Mrabet, R. Hajri, K. Hamzaoui, N. Attia Romdhane, and L. Zakraoui, "Soluble Fas and soluble Fas ligand in the joints of patients with rheumatoid arthritis and osteoarthritis: a Tunisian case-control study," Clinical and Experimental Rheumatology, vol. 25, no. 5, pp. 797-798, 2007.
[18] S. Francioli, C. Cavallo, B. Grigolo, I. Martin, and A. Barbero, "Engineered cartilage maturation regulates cytokine production and interleukin- $1 \beta$ response," Clinical Orthopaedics and Related Research, vol. 469, no. 10, pp. 2773-2784, 2011.

[19] T. Sillat, G. Barreto, P. Clarijs et al., “Toll-like receptors in human chondrocytes and osteoarthritic cartilage," Acta Orthopaedica, vol. 84, no. 6, pp. 585-592, 2013.

[20] C. Baugé, F. Legendre, S. Leclercq et al., "Interleukin-1beta impairment of transforming growth factor betal signaling by down-regulation of transforming growth factor beta receptor type II and up-regulation of Smad7 in human articular chondrocytes," Arthritis and Rheumatism, vol. 56, no. 9, pp. 30203032, 2007.

[21] N. Fukui, Y. Ikeda, T. Ohnuki et al., "Pro-inflammatory cytokine tumor necrosis factor- $\alpha$ induces bone morphogenetic protein2 in chondrocytes via mRNA stabilization and transcriptional up-regulation," The Journal of Biological Chemistry, vol. 281, no. 37, pp. 27229-27241, 2006.

[22] H. Schmal, G. M. Salzmann, E. R. Langenmair, R. Henkelmann, N. P. Südkamp, and P. Niemeyer, "Biochemical characterization of early osteoarthritis in the ankle," The Scientific World Journal, vol. 2014, Article ID 434802, 9 pages, 2014.

[23] C. M. Haslauer, K. A. Elsaid, B. C. Fleming, B. L. Proffen, V. M. Johnson, and M. M. Murray, "Loss of extracellular matrix from articular cartilage is mediated by the synovium and ligament after anterior cruciate ligament injury," Osteoarthritis and Cartilage, vol. 21, no. 12, pp. 1950-1957, 2013.

[24] B. J. Heard, N. M. Solbak, Y. Achari et al., "Changes of early posttraumatic osteoarthritis in an ovine model of simulated ACL reconstruction are associated with transient acute post-injury synovial inflammation and tissue catabolism," Osteoarthritis and Cartilage, vol. 21, no. 12, pp. 1942-1949, 2013.

[25] W. Kuon, M. Kuhne, D. H. Busch et al., "Identification of novel human aggrecan T cell epitopes in HLA-B27 transgenic mice associated with spondyloarthropathy," The Journal of Immunology, vol. 173, no. 8, pp. 4859-4866, 2004.

[26] M. Cucchiarini, M. Ekici, S. Schetting, D. Kohn, and H. Madry, "Metabolic activities and chondrogenic differentiation of human mesenchymal stem cells following recombinant adeno-associated virus-mediated gene transfer and overexpression of fibroblast growth factor 2," Tissue Engineering Part A, vol. 17, no. 15-16, pp. 1921-1933, 2011.

[27] X. Li, G. Su, J. Wang et al., "Exogenous bFGF promotes articular cartilage repair via up-regulation of multiple growth factors," Osteoarthritis and Cartilage, vol. 21, no. 10, pp. 1567-1575, 2013.

[28] A. A. Sas, Y. Jamshidi, D. Zheng et al., "The age-dependency of genetic and environmental influences on serum cytokine levels: a twin study," Cytokine, vol. 60, no. 1, pp. 108-113, 2012. 


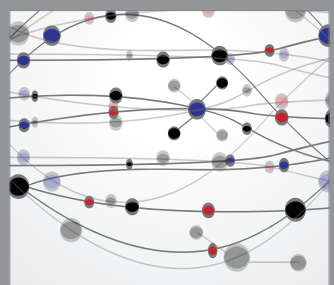

The Scientific World Journal
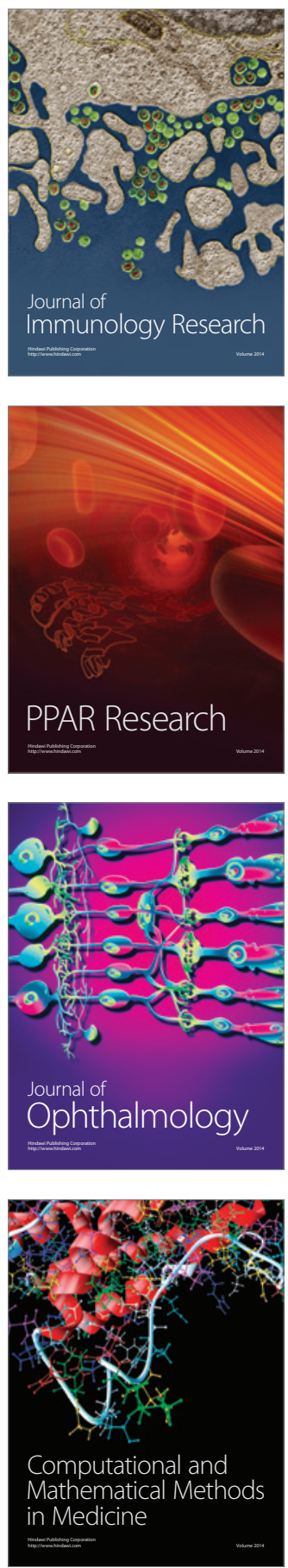

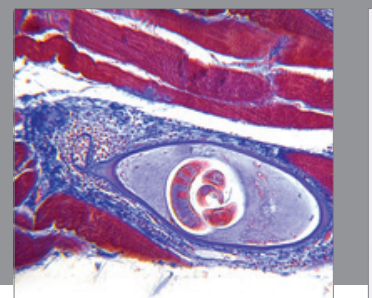

Gastroenterology

Research and Practice
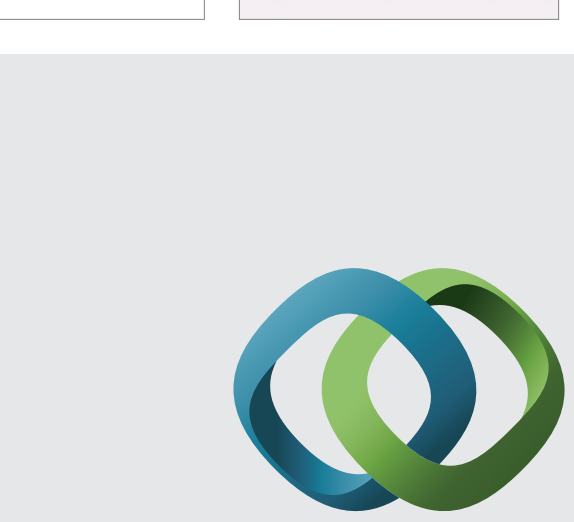

\section{Hindawi}

Submit your manuscripts at

http://www.hindawi.com
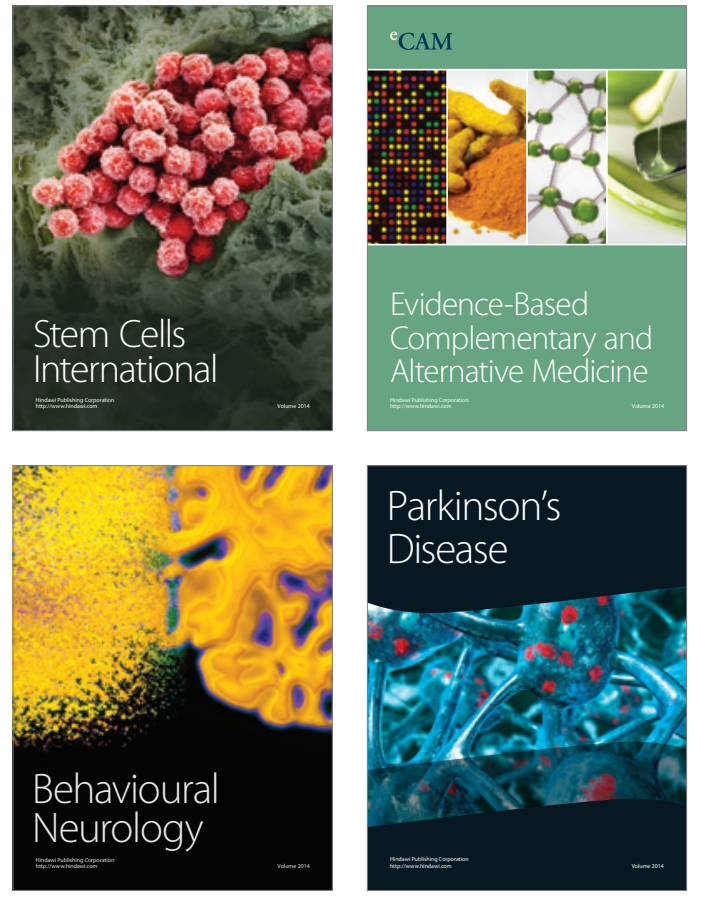
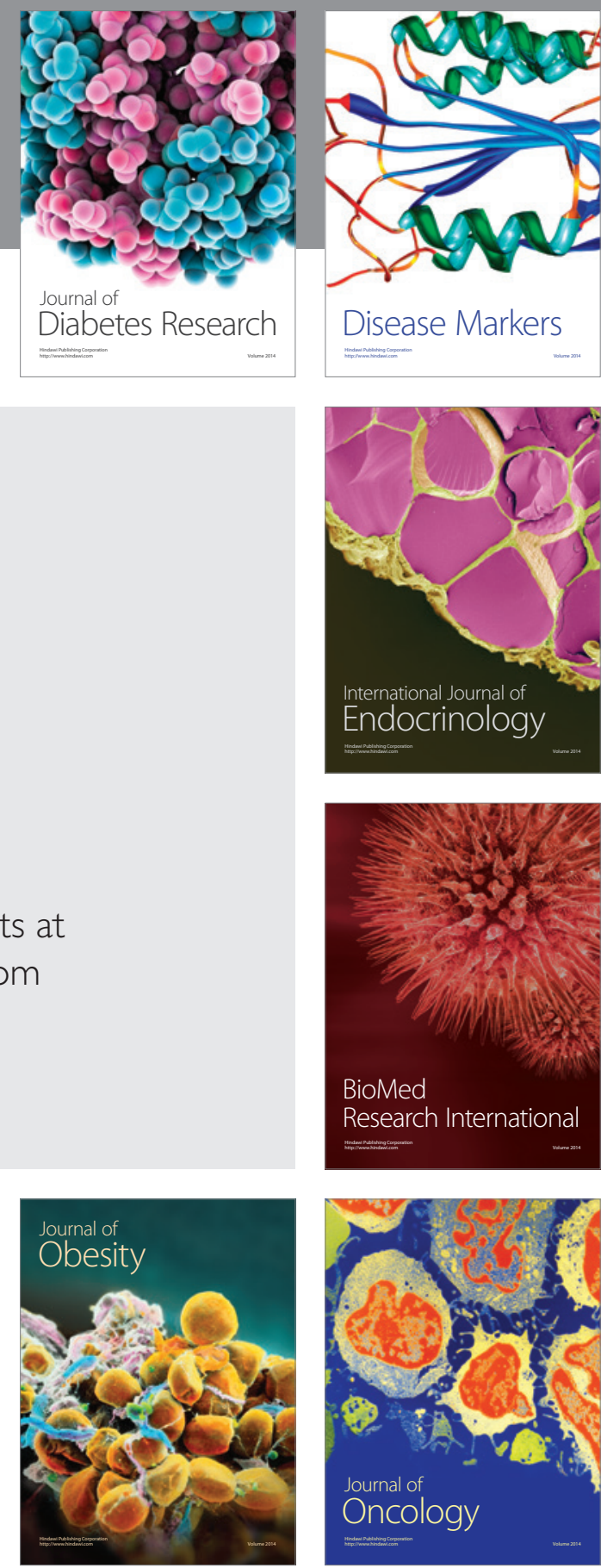

Disease Markers
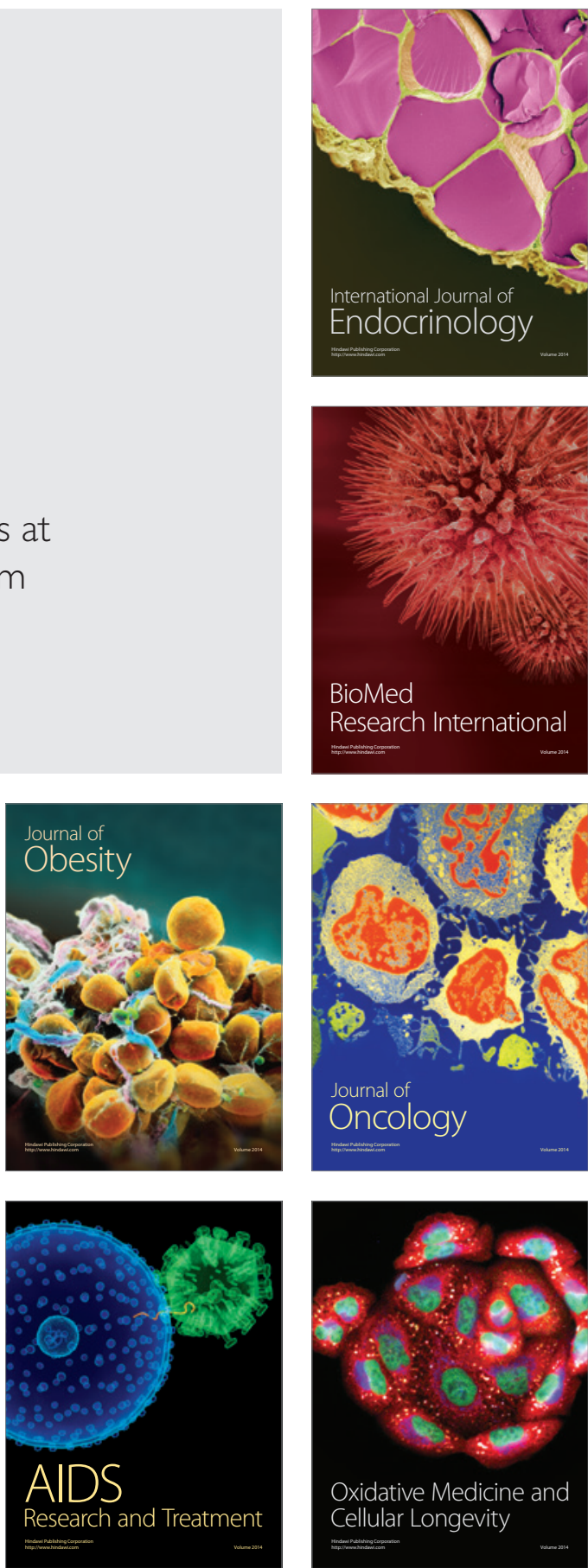